\title{
Evaluation of minimal residual disease using next-generation flow cytometry in patients with $\mathrm{AL}$ amyloidosis
}

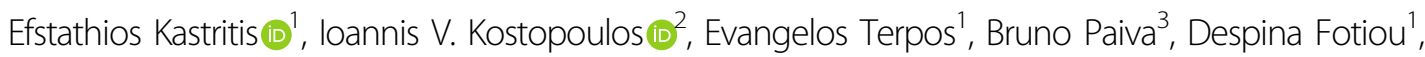 \\ Maria Gavriatopoulou', Nikolaos Kanellias', Dimitrios C. Ziogas ${ }^{1}$, Maria Roussou', Magdalini Migkou', \\ Evangelos Eleutherakis-Papaiakovou', loannis P. Trougakos ${ }^{2}$, Ourania Tsitsilonis ${ }^{2}$ and Meletios A. Dimopoulos ${ }^{1}$
}

The treatment of light chain (AL) amyloidosis aims to completely eliminate the toxic light chain production, as assessed by sensitive serum- or urine-based methods such as immunofixation and free light chain (FLCs) quantification ${ }^{1}$. Complete hematologic responses (hemCR) can be achieved in a significant proportion of patients with $\mathrm{AL}$, either with conventional therapies or with high-dose melphalan, and are associated with better overall survival and improved organ function. However, hematologic relapses still occur and organ function may continue to deteriorate due to small residual clones that may lead to disease recurrence and/or may produce very small amounts of toxic light chains which are undetectable by conventional techniques. Next-generation flow cytometry (NGF) is a very sensitive method for the evaluation of minimal residual disease (MRD) and one of the standard methods for the assessment of MRD in patients with multiple myeloma (MM), reflected in the new response assessment criteria ${ }^{2}$. Patients with MM who are negative for MRD have significantly improved progression-free and overall survival, even among those who have achieved a $\mathrm{CR}^{3,4}$. Such data are sparse in patients with $\mathrm{AL}$ amyloidosis, although the presence of MRD may prove a crucial factor for delayed organ response or deterioration of organ function despite conventional hemCR. The aim of the current study was to evaluate feasibility and

\footnotetext{
Correspondence: Efstathios Kastritis (ekastritis@med.uoa.gr)

${ }^{1}$ Department of Clinical Therapeutics, School of Medicine, National and

Kapodistrian University of Athens, Athens, Greece

${ }^{2}$ Department of Biology, School of Science, National and Kapodistrian

University of Athens, Athens, Greece

Full list of author information is available at the end of the article

These authors contributed equally: Efstathios Kastritis, loannis V. Kostopoulos.
}

applicability of MRD by NGF in patients with AL at hemCR.

We evaluated the presence of MRD in 20 patients with $\mathrm{AL}$ amyloidosis who had achieved a CR, based on negative serum and urine immunofixation, a normal FLC ratio with FLCs within normal range and a negative bone marrow (BM) biopsy ${ }^{5}$. We also evaluated five patients with normal FLCs but positive serum or urine immunofixation (i.e., at very good partial response, VGPR).

MRD was assessed in BM samples according to the Euroflow guidelines. In particular, bulk lysis was used for the osmotic lysis of erythrocytes and nucleated cells acquired were labeled using two independent eight-color panels, both containing CD19-PECy7, CD27-BV510, CD38-FITC, CD45-PerCPCy5.5, CD56-PE, and CD138BV421, with additionally CD117-APC and CD81APCC750 in the surface tube or CyIgk-APC and CyIg $\lambda$ APCC750 in the intracytoplasmic tube. Labeled antibodies were purchased from Cytognos S.L. (Salamanca, Spain), BD Biosciences (NJ, USA), and BioLegend Inc. (CA, USA). A median number of 5 million events (range: $3.9 \times 10^{6}-6.1 \times 10^{6}$ ) were acquired for each tube in a BD FACSCantoII cytometer and data analysis was conducted with Infinicyt software (Cytognos) that allowed merging of the two panels based on the six backbone markers. Therefore, aberrant plasma cells could be distinguished out of $\sim 10$ million evaluable cells per patient offering a highly sensitive approach for MRD detection, with median sensitivity level $2.3 \times 10^{-6}$ (range: $2 \times 10^{-6}-3.1 \times 10^{-6}$ ). Deploying the multiparameter nature of this assay, we confirmed the presence of all major BM subsets in all samples analyzed, thus excluding potential false-negative results due to hemodilution. 
Table 1 Baseline characteristics of AL patients in hemCR that were tested for MRD

\begin{tabular}{|c|c|c|c|}
\hline & $\begin{array}{l}\text { All patients } \\
(N=20)\end{array}$ & $\begin{array}{l}\text { MRD }^{\text {neg }}(N \\
=8)\end{array}$ & $\begin{array}{l}\mathrm{MRD}^{\text {pos }}(N= \\
12)\end{array}$ \\
\hline Age & $59(42-75)$ & $57(46-70)$ & $60(42-72)$ \\
\hline Male/female & $7 / 13$ & $3 / 5$ & $4 / 8$ \\
\hline $\mathrm{eGFR} \mathrm{ml} / \mathrm{min} / 1.73 \mathrm{~m}^{2}$ & $93(9->140)$ & 77 & 107 \\
\hline $\begin{array}{l}\mathrm{e} G \mathrm{Fr}<50 \mathrm{ml} / \mathrm{min} / \\
1.73 \mathrm{~m}^{2}\end{array}$ & 5 & 3 & 2 \\
\hline Renal involvement & $18(90 \%)$ & $8(100 \%)$ & $10(83 \%)$ \\
\hline Proteinuria gr/24 h & $7.3(4-22)$ & $9.3(5.7-22)$ & $7(4-12)$ \\
\hline dFLC (median/range) & $93(17-879)$ & $202(17-795)$ & $68(18-879)$ \\
\hline Cardiac involvement & 7 & 3 & 4 \\
\hline NTproBNP (pg/ml) & $550(30-4396)$ & $796(87-3415)$ & $346(30-4396)$ \\
\hline Mayo stage $1 / 2 / 3$ & $8 / 10 / 2$ & $3 / 4 / 1$ & $5 / 6 / 1$ \\
\hline $\mathrm{dFLC}<40 \mathrm{mg} / \mathrm{l}$ & 3 & $1 / 3(33 \%)$ & $2 / 3(67 \%)$ \\
\hline BM infiltration & $8 \%(0-30 \%)$ & $5 \%(0-20)$ & $15 \%(0-30)$ \\
\hline $\begin{array}{l}\text { Bortezomib-based } \\
\text { induction }\end{array}$ & $17(85 \%)$ & 7 & 10 \\
\hline MDex & $3(15 \%)$ & 1 & 2 \\
\hline $\begin{array}{l}\text { Consolidation with } \\
\text { HDM/ASCT }\end{array}$ & $8(40 \%)$ & $2 / 8(25 \%)$ & 6/8 (75\%) \\
\hline Any organ response & $14(70 \%)$ & $6 / 8(75 \%)$ & $8 / 12(67 \%)$ \\
\hline Renal response & $12 / 18(67 \%)$ & $6 / 8(75 \%)$ & $6 / 10(60 \%)$ \\
\hline Cardiac response & $4 / 7$ & $3 / 3(100 \%)$ & $1 / 4(25 \%)$ \\
\hline Liver response & $2 / 3$ & $1 / 1(100 \%)$ & $1 / 2(50 \%)$ \\
\hline
\end{tabular}

The median age of the patients at the time of diagnosis was 59 years (range: $42-75$ ), $74 \%$ had lambda light chain $\mathrm{AL}, 90 \%$ had renal, $15 \%$ had liver, and $35 \%$ had cardiac involvement; $40 \%$ were Mayo stage- $1,50 \%$ stage-2, and $10 \%$ stage- 3 . At the time of diagnosis, median dFLC was $93 \mathrm{mg} / \mathrm{l}$ (range: 17-879) and four (20\%) had negative serum and urine immunofixation; median BM infiltration by clonal plasma cells was $8 \%$. Primary treatment was bortezomib-based in $85 \%$ and melphalan/dexamethasone (MDex) in 15\%, while $40 \%$ have received high-dose melphalan with ASCT as consolidation. At the time of MRD testing, 9/18 (50\%) patients had achieved a renal response, 4/7 (57\%) patients with cardiac involvement had a cardiac response, and 2/3 (67\%) a liver response.

Eight (40\%) patients were negative for the presence of MRD (MRD $\left.{ }^{\text {neg }}\right)$ and $12(60 \%)$ were positive $\left(\mathrm{MRD}^{\mathrm{pos}}\right)$. Notably, 5/12 (42\%) MRD ${ }^{\text {pos }}$ cases had a very low residual tumor load at level $<3 \times 10^{-5}$ (in two cases aberrant cells were detected at levels between $10^{-5}$ and $10^{-6}$ ). The median time from hemCR to MRD testing was 36 months
(39 months for $M R D^{\text {pos }}$ vs 35 months for $M R D^{\text {neg }}$ patients). MRD was positive in $3 / 4$ patients who had negative immunofixation in the serum and urine and in $2 /$ 3 patients with $\mathrm{dFLC}<40 \mathrm{mg} / \mathrm{l}$ at the time of diagnosis. In contrast all patients in VGPR that were tested were MRD $^{\text {pos }}$

Organ responses of at least one involved organ were documented in 14/20 (70\%) patients in CR and in particular in 8/12 (67\%) of patients with $\mathrm{MRD}^{\text {pos }}$ vs $6 / 8(75 \%)$ of patients with $\mathrm{MRD}^{\text {neg }}$ disease. Among cardiac responders $(n=4)$, three were $M_{R D} D^{\text {neg }}$ and one was MRD ${ }^{\text {pos }}$. Renal responses were 6/9 (67\%) in $\mathrm{MRD}^{\text {pos }}$ and $6 / 8(75 \%)$ in $M_{R} D^{\text {neg }}$ patients. Among $M^{2} D^{\text {neg }}$ patients, $3 / 8$ had response in more than one organ (both had a cardiac and a renal response); among $\mathrm{MRD}^{\text {pos }}$ patients all had responses to a single organ.

We then analyzed for possible differences in the baseline characteristics of those in CR that achieved vs those that did not achieve MRD negativity; we found no significant differences in baseline characteristics such as age, gender, serum FLC levels or dFLC, BM infiltration by plasma cells, NTproBNP levels, or Mayo stage (all $p$ values $>0.5)$. Among patients who received ASCT, 2/8 (25\%) were $\mathrm{MRD}^{\text {neg }}$ vs $6 / 12(50 \%) \mathrm{MRD}^{\text {neg }}$ among patients who did not have ASCT as part of their primary therapy $(p=$ 0.264). Of the three patients treated with MDex, one (33\%) was found $M^{2} D^{\text {neg }}$. A summary of all available baseline characteristics in MRD ${ }^{\text {pos }}$ vs MRD ${ }^{\text {neg }}$ patients of our cohort are provided in Table 1 . Thus, we could not identify possible baseline factors associated with a higher probability of $M R D^{\text {neg }}$, in this highly selected population that includes patients with low or intermediate risk disease, who achieved a hemCR and who have already had a long survival associated with high rates of organ responses.

This is the first report on MRD evaluation in patients with AL amyloidosis, using NGF, with high sensitivity levels approaching $10^{-6}$. Importantly, in $5 / 12 \mathrm{MRD}^{\text {pos }}$ cases residual clonal plasma cells would have been undetectable if a lower level of sensitivity had been used (Fig. 1) and as a consequence, these patients would have been considered as $\mathrm{MRD}^{\text {neg }}$, indicating the importance of high sensitivity methods for MRD assessment. It is also important that among patients with very low levels of detectable clonal light chains at the time of diagnosis we were able to identify MRD after treatment. Thus, NGF may also be a useful method for the detection of clonal disease in patients with very low burden of light chains which may be difficult to detect but which may also be quite toxic, as suggested by previous studies using lesssensitive flow cytometry methods ${ }^{6,7}$.

In $\mathrm{AL}$ amyloidosis the plasma cell clone is usually modest in size and usually lacks high-risk cytogenetics such as del17p or $\mathrm{t}(4 ; 14)^{8,9}$. Treatment with bortezomib- 

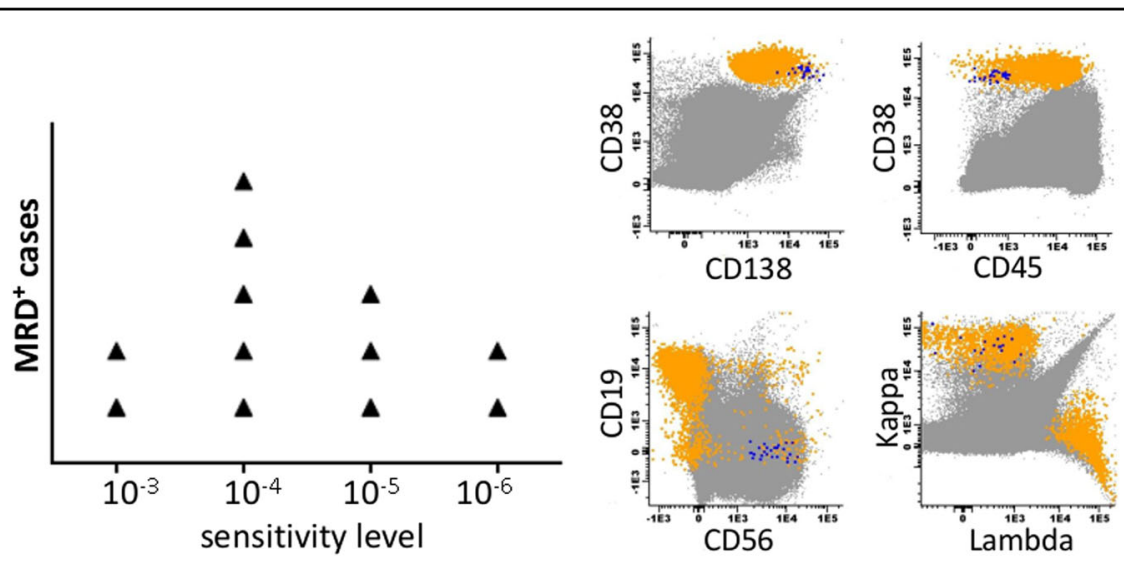

Fig. 1 MRD detection of AL patients using next-generation flow cytometry (NGF). Left panel: The 12 MRD + AL cases of our cohort according to the detection level of aberrant plasma cells (APCs). Right panel: A phenotypic analysis of MRD + case at the level of $10^{-6}$. APCs (showing in blue) have a clearly distinct phenotype from normal plasma cells (shown in orange). The rest of the bone marrow nucleated cells are shown in gray

based therapy or with high or conventional dose melphalan results in high response rates and deep responses. Thus, despite the high depth of detecting aberrant plasma cells in our setting, it would be expected that more patients in hemCR would be MRD ${ }^{\text {neg }}$. Our results, however, indicate that more than half of AL patients in hemCR (by conventional methods) are MRD positive (60\%). The implications of this observation may be significant, especially if confirmed in larger series. The presence of MRD may be associated with a higher chance of hematologic relapse and subsequent organ function deterioration, necessitating therapy. Moreover, in MRDpositive patients the minimal amount of the toxic light chains produced by residual clonal cells may delay or undermine the restoration of organ function, or, may lead to further organ function deterioration. Conversely, MRD negativity may be associated with deeper organ responses, with responses in more "sensitive" organs, such as the heart, and with responses in more than one organ. The small number of patients in our study does not allow for firm conclusions however, it is notable that among cardiac responders, 3/4 were MRD negative. Another aspect underscored by our results is the relatively low incidence of MRD negativity among patients who had ASCT, which highlights concerns about the need of further consolidation or maintenance after ASCT in patients with AL, similar to myeloma. Similarly, a recent study by Lee et al. ${ }^{10}$ reported that $2 / 5(40 \%) \mathrm{AL}$ amyloid patients after ASCT were $M R D^{\text {neg }}$. The introduction of novel strategies such as monoclonal antibodies targeting CD38 (such as daratumumab ${ }^{11-13}$ ) or those targeting the amyloid fibrils ${ }^{14}$ will change the potential options for patients that remain MRD positive.

In conclusion, among patients with AL amyloidosis in sustained hemCR, $40 \%$ were $\mathrm{MRD}^{\text {neg }}$ and $60 \%$ were $\mathrm{MRD}^{\text {pos }}$, as assessed with high sensitivity NGF. These findings may have implications in the management of patients with AL who achieve a hemCR, especially for patients who fail to achieve an organ response and may also have implications for their management, in an era of expanding treatment options.

\section{Acknowledgements \\ This study was supported by the Hellenic Society of Medical Oncology (HeSMO) and also supported internationally by the Black Swan Research Initiative of the International Myeloma Foundation, and the Spanish Instituto de Salud Carlos III/Subdirección General de Investigación Sanitaria (FIS: PI13/ 02196).}

\section{Author details}

'Department of Clinical Therapeutics, School of Medicine, National and Kapodistrian University of Athens, Athens, Greece. ${ }^{2}$ Department of Biology, School of Science, National and Kapodistrian University of Athens, Athens, Greece. ${ }^{3}$ Centro de Investigación Médica Aplicada, Clinica Universidad de Navarra, IDISNA, Pamplona, Spain

\section{Conflict of interest}

MAD has received honoraria from Amgen, Celgene, Janssen, BMS, Takeda, ET has received honoraria from Amgen, Genesis Pharma, Takeda, E.K. has received honoraria and travel grants from Amgen, Genesis Pharma, Janssen, Takeda, and Prothena. The remaining authors declare that they have no conflict of interest.

\section{Publisher's note}

Springer Nature remains neutral with regard to jurisdictional claims in published maps and institutional affiliations.

Received: 19 December 2017 Revised: 16 March 2018 Accepted: 10 April 2018

Published online: 24 May 2018

\section{References}

1. Kastritis, E. \& Dimopoulos, M. A. Recent advances in the management of AL amyloidosis. Br. J. Haematol. 172, 170-186 (2016).

2. Kumar, S. et al. International Myeloma Working Group consensus criteria for response and minimal residual disease assessment in multiple myeloma. Lancet Oncol. 17, e328-e346 (2016). 
3. Flores-Montero, J. et al. Next generation flow for highly sensitive and standardized detection of minimal residual disease in multiple myeloma. Leukemia 31, 2094-2103 (2017).

4. Paiva, B. et al. Minimal residual disease monitoring and immune profiling in multiple myeloma in elderly patients. Blood 127, 3165-3174 (2016).

5. Palladini, G. et al. New criteria for response to treatment in immunoglobulin light chain amyloidosis based on free light chain measurement and cardiac biomarkers: impact on survival outcomes. J. Clin. Oncol. 30, 4541-4549 (2012).

6. Paiva, B. et al. The clinical utility and prognostic value of multiparameter flow cytometry immunophenotyping in light-chain amyloidosis. Blood $\mathbf{1 1 7}$ 3613-3616 (2011)

7. Paiva, B. et al. Phenotypic, transcriptomic, and genomic features of clonal plasma cells in light-chain amyloidosis. Blood 127, 3035-3039 (2016).

8. Bochtler, T. et al. Translocation $t(11 ; 14)$ is associated with adverse outcome in patients with newly diagnosed $\mathrm{AL}$ amyloidosis when treated with bortezomib-based regimens. J. Clin. Oncol. 33, 1371-1378 (2015).
9. Bochtler, T. et al. Gain of chromosome 1q21 is an independent adverse prognostic factor in light chain amyloidosis patients treated with melphalan/ dexamethasone. Amyloid 21, 9-17 (2014).

10. Lee, $\mathrm{H}$. et al. Minimal residual disease (MRD) assessment by flow cytometry after ASCT for AL amyloidosis: are we there yet? Bone Marrow Transplant. 52, 915-917 (2017)

11. Popat, R. et al. Real world data of the impact of first cycle daratumumab on multiple myeloma and AL amyloidosis services. Br. J. Haematol. (2017) https:// doi.org/10.1111/bjh.14897.

12. Kaufman, G. P. et al. Daratumumab yields rapid and deep hematologic responses in patients with heavily pretreated AL amyloidosis. Blood 130, 900-902 (2017).

13. Sher, T., Fenton, B., Akhtar, A. \& Gertz, M. A. First report of safety and efficacy of daratumumab in 2 cases of advanced immunoglobulin light chain amyloidosis. Blood 128, 1987-1989 (2016).

14. Gertz, M. A. et al. First-in-human phase $1 / 1 /$ study of NEOD001 in patients with light chain amyloidosis and persistent organ dysfunction. J. Clin. Oncol. 34, 1097-1103 (2016) 